$\underline{\text { Paper }}$

Computational Physics and Engineering Division(10)

\title{
Sensitivity and Uncertainty Analysis for Nuclear Criticality Safety Using KENO in the SCALE Code System
}

\author{
Bradley T. Rearden \\ Oak Ridge National Laboratory, \\ P. O. Box 2008, \\ Oak Ridge, TN 37831-6370 \\ (865) 574-6085 office number \\ (865) 576-3513 fax number \\ reardenbt@ornl.gov
}

Submitted to the

Monte Carlo 2000 C Advanced Monte Carlo Radiation Physics,

Particle Transport Simulation Applications,

October 23B26, 2000,

Lisbon, Portugal

The submitted manuscript has been authored by a contractor of the U.S. Government under contract No. DEAC05-00OR22725. Accordingly, the U.S. Government retains a nonexclusive, royalty-free license to publish or reproduce the published form of this contribution, or allow others to do so, for U.S. Government purposes.

*Managed by UT-Battelle, LLC, under contract DE-AC05-00OR22725 with the U.S. Department of Energy. 


\title{
Sensitivity and Uncertainty Analysis for Nuclear Criticality Safety Using KENO in the SCALE Code System
}

\author{
Bradley T. Rearden \\ Oak Ridge National Laboratory, P.O. Box 2008, M.S. 6370, Oak Ridge, TN 37831- \\ 6370, USA, Phone (865) 574-6085, Fax (865) 576-3513, email reardenbt@ ornl.gov
}

\begin{abstract}
Sensitivity and uncertainty methods have been developed to aid in the establishment of areas of applicability and validation of computer codes and nuclear data for nuclear criticality safety studies. A key component in this work is the generation of sensitivity and uncertainty parameters for typically several hundred benchmarks experiments used in validation exercises. Previously, only onedimensional sensitivity tools were available for this task, which necessitated the remodeling of multidimensional inputs in order for such an analysis to be performed. This paper describes the development of the SEN3 Monte Carlo based sensitivity analysis sequence for SCALE.

Two options in the SEN3 package for the reconstruction of angular-dependent forward and adjoint fluxes are described and contrasted. These options are the direct calculation of flux moments versus the calculation of angular fluxes, with subsequent conversion to flux moments prior to sensitivity coefficient generation. The latter technique is found to be significantly more efficient.
\end{abstract}

\section{Introduction}

Extensive work has been conducted recently to demonstrate that sensitivity and uncertainty $(\mathrm{S} / \mathrm{U})$ analysis methodologies can be used to help establish areas of applicability and the related validation of computational codes and data for nuclear criticality safety[1]. In this work, three methodologies were demonstrated to quantitatively establish areas of applicability and to establish code and data biases. The methodologies are based on integral parameter applications, uncertainty analysis theory and generalized linear-least-squares methodology (GLLSM). In each of the three methodologies, energy-dependent sensitivity profiles for multiple nuclides and reactions are required to perform the analyses. These sensitivities predict the relative change in the system $k_{\text {eff }}$ due to a perturbation in a constituent cross section. Recently, the differential approach to perturbation theory as implemented in the FORSS[2] system in the 1970s was updated and applied to the industry-standard SCALE[3] code system for one-dimensional (1-D) and three-dimensional (3-D) criticality safety analyses. The perturbation techniques have been implemented in two prototypic analysis sequences, which execute a series of SCALE's functional modules in a specified order. The SEN1 sequence uses 1-D deterministic neutron transport to calculate the forward and adjoint neutron fluxes necessary for the sensitivity coefficient 
generation [4]. The prototypic SEN3[5] sequence utilizes 3-D Monte Carlo techniques from an enhanced version of the KENO V.a code to generate the necessary forward and adjoint fluxes and computes sensitivity coefficients and their associated uncertainties. Recent enhancements in the SEN3 analysis sequence are the focus of this paper.

\section{Methods}

The SEN3 sequence implements an enhanced version of the KENO V.a Monte Carlo code as well as the neutron cross-section processing modules of SCALE and the newly created Sensitivity Analysis Module for SCALE (SAMS). The enhanced version of KENO V.a, which was specifically developed for use in sensitivity analyses, is used to generate the forward and adjoint angular neutron fluxes, as well as flux moments, which are necessary for the generation of sensitivity coefficients. A novel approach to the calculation of the appropriate neutron flux moments was designed specifically for this project.

\subsection{Calculation of Flux Moments}

In previous publications on this topic, the techniques whereby the flux moments were calculated directly in KENO V.a were presented[5,6]. Recently, these techniques have been updated to improve the efficiency of the calculation by calculating the angular neutron flux in KENOV.a and, subsequently, by performing a spherical harmonics expansion within the SAMS module to produce the necessary flux moments.

Using the track length estimator method, the group-wise scalar flux within a single region for a single generation of particles is calculated as follows [7,8,9]:

$$
\phi_{g, z}=\frac{\sum_{j=1}^{J} u t_{j, z} l_{j, z}}{V_{z} \sum_{j=1}^{J} u t_{j, 0}}
$$

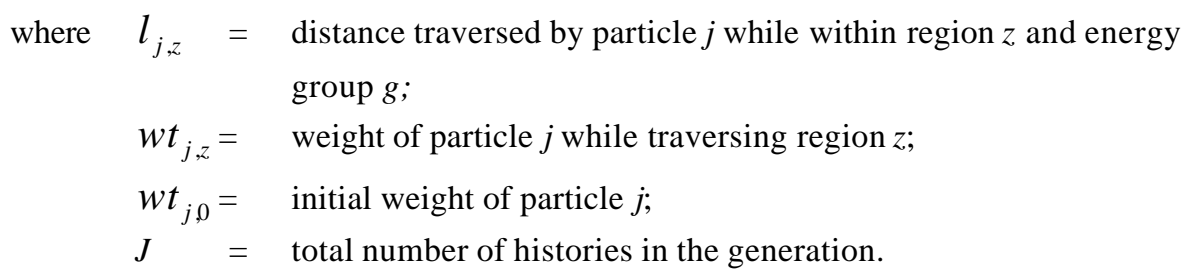

This method is easily modified to calculate the group-wise angular flux in a region as 


$$
\phi_{g, k}^{n}=\frac{\sum_{j=1}^{J} w t_{j, z} l_{j, z, n}}{V_{z} \sum_{j=1}^{J} w t_{j, 0}}
$$

where $l_{j, z, n}=$ the distance traversed by particle $j$ while within region $z$ and energy group $g$ within the solid angle $d \hat{\Omega}_{n}$ about the quadrature direction $d \hat{\Omega}_{n}$.

In Eq. (2) the direction of travel for each history is calculated using a transformed coordinate system based on a polar, rather than Cartesian, position vector. When combined with the spherical harmonics expansion, which is performed after the Monte Carlo calculations are complete, this is a 3-D extension of the 1-D method for calculating the flux moments in terms of Legendre polynomials based only on $\mu$ the direction cosine with respect to the spatial coordinate.

For the angular flux tally, the direction of travel of the particle is calculated in a transformed coordinate system such that the transformed polar or $\hat{k}^{\prime}$ axis is co-linear with the position vector $\vec{r}_{c}$ directed from the centroid of the system to the point at which the flux tally occurs. The centroid must be used because KENO V.a geometry can be input relative to any reference point. By using the centroid as a reference point, the consistency of the moment calculation is ensured with differing models of the same system. The actual position and direction of travel of the particle remain unchanged, but the angular fluxes are tallied using this transformed coordinate system. This coordinate transform is illustrated in Fig. 1. Here, $\hat{i}, \hat{j}$, and $\hat{k}^{\prime}$ represent the directional coordinate system axes; $\mu, \eta$, and $\xi$, represent the direction cosines; and $\theta$ and $\rho$ represent the polar and azimuthal angles of the "normal" coordinate system. The same symbols "primed" represent the transformed coordinate system. With the direction cosines consistently transformed for each history, the new polar and azimuthal angles can be computed and each history can be appropriately tallied for the flux moment calculation by spherical harmonics expansion. The enhanced KENO V.a code uses symmetric-level quadrature sets up to an order of $S_{16}$. The SAMS module will perform a spherical harmonics expansion up to third-order. 


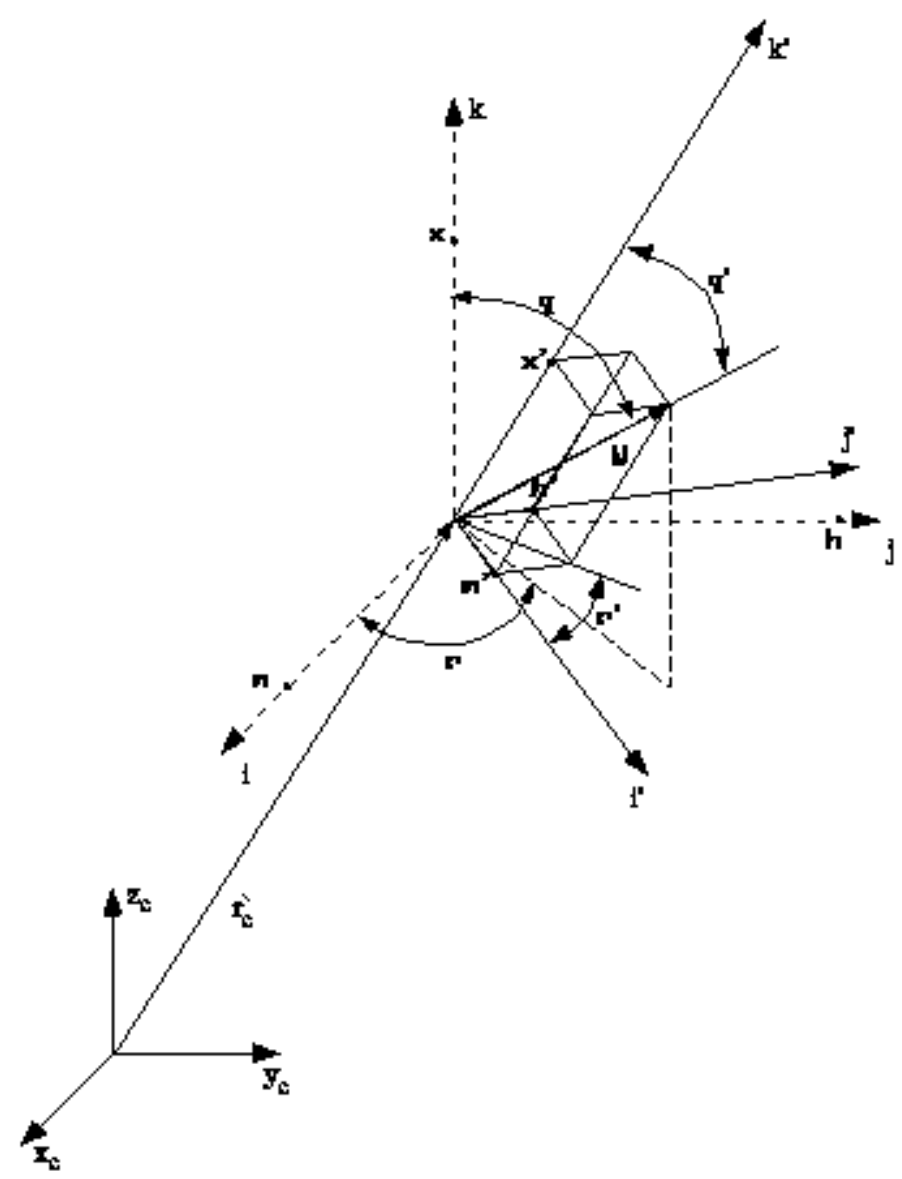

Fig. 1. Coordinate system transform for angular flux tally

\subsection{Sensitivity Coefficient Generation}

Once the fluxes have been calculated, the SAMS module, which is executed within the SEN3 sequence, automatically selects all of the sensitivity parameters that can be calculated for each nuclide in each region of the system based on available crosssection data. Sensitivity parameters for a given nuclide may be generated for a number of parameters, including total, scatter, capture, and fission cross sections, as well as $\bar{v}$ and $\chi$. The sensitivities for any nuclide-reaction pair calculated with SEN3 are output on three bases: group-wise-region-dependent, energy-integrated-region-dependent, and energy- and region-integrated. This method gives the user the ability to interpret the data with varying levels of detail. SEN3 produces sensitivities in a number of 
convenient formats for further analysis, either manually or with other automated techniques. SEN3 produces the data necessary for evaluations with the integral parameter applications and cross-section covariance theory as well as GLLSM. Energy-dependent sensitivity profiles and their associated Monte Carlo uncertainties generated by SAMS can be visually displayed using the SAMSPLOT program. The sensitivity coefficient generation is fully explained in [5] and [6].

\section{Results}

The SEN3 sequence has been applied to hundreds of critical benchmark experiments and several practical design applications. This work as been documented elsewhere[10] and will not be repeated here. Instead, the results of the new angular flux method for calculating the flux moments are presented. To verify the accuracy and efficiency of this method, an early test case for this code was repeated. The selected sample problem is based on an unreflected rectangular parallelpiped containing a homogeneous mixture $\mathrm{UF}_{4}$ and paraffin with an enrichment of $2 \%$ in ${ }^{235} \mathrm{U}$. The $\mathrm{H} /{ }^{235} \mathrm{U}$ atomic ratio is $293.9: 1$. The dimensions of the experiment were $56.22 \mathrm{~cm} \times 56.22 \mathrm{~cm} \times 122.47 \mathrm{~cm}[11]$. This critical experiment is designated as CAS14. For the purposes of an accurate comparison with the SEN1 code, this experiment was modeled as a sphere of the mixture material with a critical radius of $38.50 \mathrm{~cm}$.

Two executions of SEN3 were conducted for this experiment: The first used the original flux moment tally method conducted during the Monte Carlo execution. The second used the new angular flux tally within KENO V.a and the subsequent flux moment calculation within the SAMS module. The parameters for the SEN3 executions are shown in Table 1. The sensitivity results for SEN1 and the two cases from SEN3 are shown in Table 2 The parameters shown in Table 1 demonstrate that the problem executes in nearly half the time using the new methodology, and the results shown in Table 2 demonstrate that virtually identical results are produced by all methods.

Table 1. Parameters from SEN3 executions for CAS14

\begin{tabular}{lcccc}
\hline Parameter & $\begin{array}{c}\text { Flux } \\
\text { moments } \\
\text { (forward) }\end{array}$ & $\begin{array}{c}\text { Flux } \\
\text { moments } \\
\text { (adjoint) }\end{array}$ & $\begin{array}{c}\text { Angular } \\
\text { fluxes } \\
\text { (forward) }\end{array}$ & $\begin{array}{c}\text { Angular } \\
\text { fluxes } \\
\text { (adjoint) }\end{array}$ \\
\hline $\begin{array}{c}\text { Neutrons per } \\
\text { generation } \\
\text { Generations }\end{array}$ & 1500 & 4500 & 1500 & 4500 \\
$\begin{array}{c}\text { Legendre order } \\
\text { for flux moments }\end{array}$ & 400 & 400 & 400 & 400 \\
$\begin{array}{c}\text { Angular quadrature } \\
\text { Execution time } \\
\text { (CPU minutes) }\end{array}$ & $\mathrm{P}_{3}$ & $\mathrm{P}_{3}$ & $\mathrm{P}_{3}$ & $\mathrm{P}_{3}$ \\
\hline
\end{tabular}


Table 2. Energy-integrated sensitivities for spherical models of CAS14

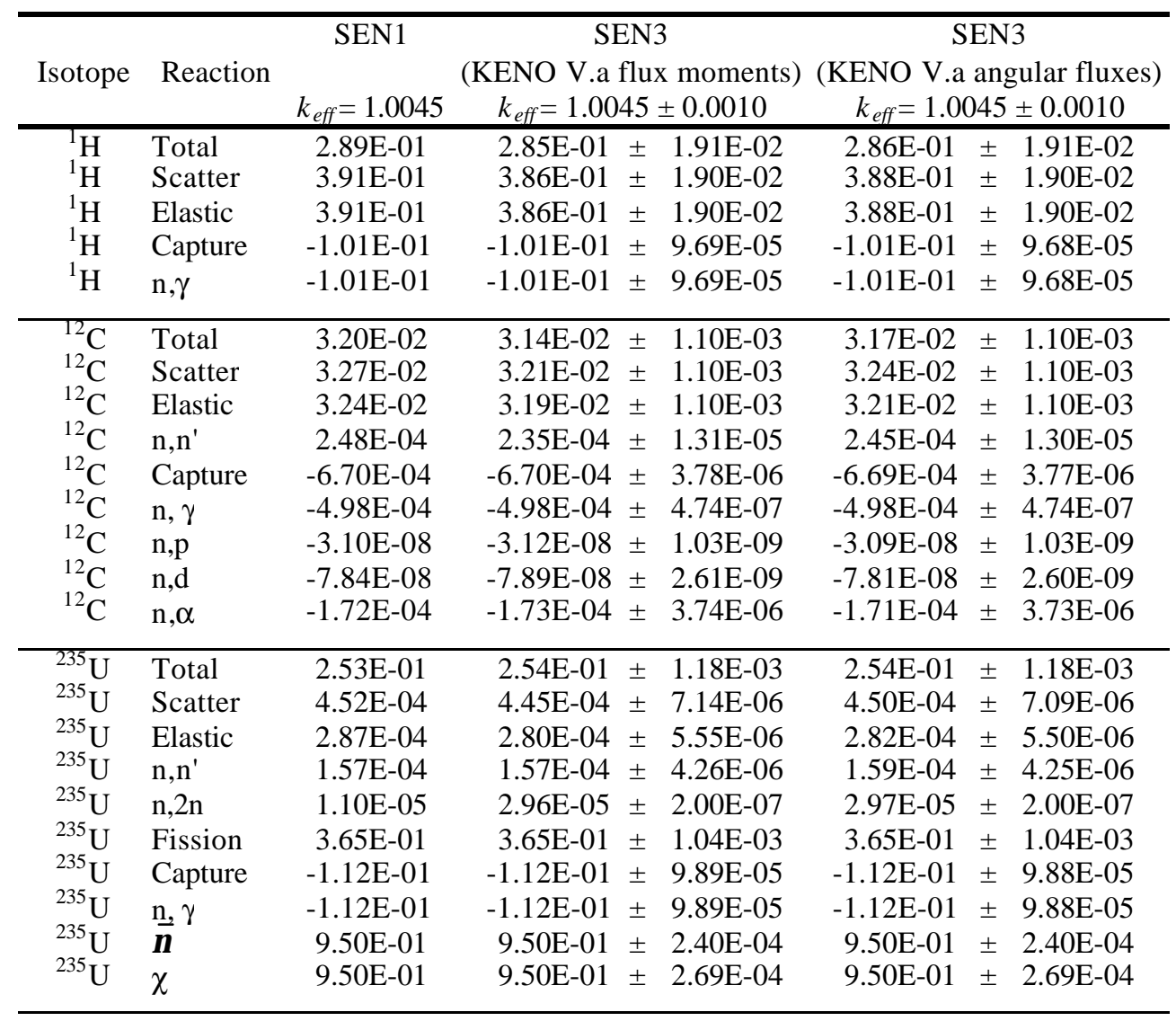

\section{Conclusions}

With the development of SEN3, a valuable tool has been added to those previously available at ORNL for determining the range of applicability of critical experiments. The calculation of sensitivity parameters from existing 3-D CSAS25 experiment models permits the use of the range-of-applicability techniques for a broader range of critical experiments and proposed criticality applications. However, further development is necessary before these techniques are available in a production-level code. Although the new methodology for the calculation of the flux moments produces a considerable speed enhancement over the previous methodology, shortcomings to the sensitivity methods in general, as noted in previous publications [5,6], have not yet been fully addressed. 


\section{References}

1. B. L. Broadhead, C. M. Hopper, R. L. Childs, and C. V. Parks: Sensitivity and Uncertainty Analyses Applied to Criticality Validation, Volume 1: Methods Development, NUREG/CR-5593, Vol. 1 (ORNL/TM-13692/V1), U.S. Nuclear Regulatory Commission, Oak Ridge National Laboratory (1999).

2. J. L. Lucius et al.: A Users Manual for the FORSS Sensitivity and Uncertainty Analysis Code System, ORNL-5316, Union Carbide Corp., OakRidge National Laboratory (1981).

3. SCALE: A Modular Code System for Performing Standardized Computer Analyses for Licensing Evaluation, NUREG/CR-0200, Rev. 6 (ORNL/NUREG/CSD-2R6), Vols. I, II, and III, May 2000. Available from Radiation Safety Information Computational Center at OakRidge National Laboratory as CCC-545.

4. R. L. Childs: SEN1: A One Dimensional Cross-Section Sensitivity and Uncertainty Module for Criticality Safety Analysis, NUREG/CR-5719 (ORNL/TM-13738), U.S. Nuclear Regulatory Commission, OakRidge National Laboratory (1999).

5. B. T. Rearden: "Development of SAMS: A Sensitivity Analysis Module for the SCALE Code System Using KENO V.a in the CSAS25 Sequence," Ph.D. Dissertation, Texas A\&M University (1999).

6. B. T. Rearden: "SAMS: A Sensitivity Analysis Module for Criticality Safety Analysis Using Monte Carlo Techniques," Proc. of PHYSOR 2000, ANS Int. Topical Meeting on Advances in Reactor Physics and Mathematics and Computation into the Next Millennium, CD-ROM, Pittsburgh, Pennsylvania, May 7-12, 2000, ANS

7. E. E. Lewis and W. F. Miller, Jr.: "Computational Methods of Neutron Transport," American Nuclear Society, La Grange Park, IL (1993).

8. L. L. Carter and E. D. Cashwell: "Particle-Transport Simulation with the Monte Carlo Method," TID-26607, U.S. Energy Research and Development Administration (1975).

9. E. L. Redmond II: "Multigroup Cross Sections Generation Via Monte Carlo Methods," Ph.D. Dissertation, Massachusetts Institute of Technology (June 1997).

10. B. T. Rearden, C. M. Hopper, K. R. Elam, B. L. Broadhead, and P. B. Fox: "Prototypic Applications of Sensitivity and Uncertainty Analysis for Experiment Needs," Proc. of ANS/ENS 2000 International Winter Meeting and Embedded Topical Meetings, CD-ROM, November 12-16, 2000, Washington, D.C.

11. W. C. Jordan, N. F. Landers, and L. M. Petrie: Validation of KENO V.a Comparison with Critical Experiments, ORNL/CSD/TM-238, Martin Marietta Energy Systems, Oak Ridge National Laboratory (December 1986). 